\section{Laser path planning of burn-out rule for LOM process}

\author{
Y.Y. Chiu and \\ Y.S. Liao
}

\section{The authors}

Y.Y. Chiu is based at the Department of Industrial Design, Oriental Institute of Technology, Taiwan, Republic of China.

Y.S. Liao is based at the Department of Mechanical Engineering, National Taiwan University, Taiwan, Republic of China.

\section{Keywords}

Lasers, Layered manufacturing

\section{Abstract}

The waste material removal process in the laminated object manufacturing, so-called de-cubing, is a time-consuming work resulting from the inherent adhesion between the part and the waste material. A new method to improve the problem is proposed in this paper. It is noticed that when the temperature of the working area, being raised by the laser beams, reaches the "decomposition temperature" of the adhesive, the stickiness of the adhesive will "burn out". Based on these findings, a novel laser path planning strategy is introduced. The threshold value of the slope of the part which determines whether the burning-out rule should be applied in laser path planning is derived. If the slope of the part falls below the threshold value, the burning-out rule is utilized. Under such circumstances, an optimal laser path according to the proposed thermomechanical model is designated to the overlap zone to burn out the binder. Experimental results indicate that the method increases the de-cubing efficiency, reduces the de-cubing time required and damage the part less likely.

\section{Electronic access}

The Emerald Research Register for this journal is available at

http://www.emeraldinsight.com/researchregister

The current issue and full text archive of this journal is available at http://www.emeraldinsight.com/1355-2546.htm

Rapid Prototyping Journal

Volume $9 \cdot$ Number $4 \cdot 2003 \cdot$ pp. 201-211

(C) MCB UP Limited · ISSN 1355-2546

DOI 10.1108/13552540310489587

\section{Introduction}

Rapid prototyping is a new class of automated fabrication process that allows the construction of three-dimensional objects from solid or surface CAD files by horizontally sectioning the desired model into slices and then constructing each cross-section from the bottom up layer one at a time. Laminated object manufacturing (LOM) is one of the most effective rapid prototyping techniques in terms of speed and cost effectiveness. It uses the "bond-then-cut" principle. A sheet is laminated to the previously laid and bonded layers by a hot roller. The roller applies heat and pressure as it is rolling over the sheet, which has a thin layer of thermoplastic adhesive on the down-facing surface. After the new layer is bonded, a focused beam of $\mathrm{CO}_{2}$ laser incises the outline of the part. Laser power is adjusted to cut through only one layer of lamination. The unused material is left in place, but is, diced with crosshatch into small pieces called "tiles" for easy removal. The iterative process of bonding and laser cutting is repeated until the construction of the final layer is completed. Once all layers have been laminated and cut, waste material is removed, so called de-cubing, to expose the finished part.

As far as LOM is concerned, the adhesive strength of the waste material is the same as that of the part (Sonmez and Hahn, 1998). Owing to the inherent adhesion between the part and the waste material, one has to take extra care in de-cubing so as to make sure that the waste material is removed without damaging the geometric shape of the part. In other words, de-cubing is a time-consuming, labor intensive and experience dependent work. Thus, the improvement of the adhesion at the contact area between the part and the waste material will contribute positively to the efficiency of de-cubing.

A modified LOM system is developed for manufacturing ceramic part. (Klosterman et al., 1996, 1997a, b, c). The brittleness of ceramic material makes de-cubing difficult. To solve the problem of waste removal, they used the adaptive crosshatch method, which effectively reduced the bonding strength between the part and the waste. Klosterman and his co-workers contended that the differences in the bonding strength 
between the part and the waste determines the difficulty of de-cubing. For the overlap zone of the part and waste between the two successive layers, the contact area is reduced and the bonding strength between the part and the waste is lowered by employing fine crosshatch. However, since the adaptive crosshatch method repeats the working path in the overlap zone and reduces the part accuracy, it is not a very efficient method.

A lamination method that could achieve selective adhesion process, coined "selective adhesive and hot process (SAHP)", was developed (Inui et al., 1994). The method is based on the principle of a photocopy machine. The paper is divided into two regions namely, the part region and the waste region. An adhesive is coated on the whole part region, but for the waste region it applies only to the mesh grid. Then, a hot roller is used to exert pressure and heat to integrate the adhesive and paper. Then, a knife is used to cut according to the contour of part cross-section and mesh grid. These steps are repeated until the construction of the final layer is completed. This approach has the advantage of substantially improving the post-processing issues in de-cubing. The disadvantage is that the dissipation of adhesive coating expanded the adhesive region to larger than expected. As the region expands along the part contour to the waste region, it causes more problems for de-cubing. Besides, the adhesive coating on line also weakens the part strength, which compromises the mechanical qualities to be inferior to those of LOM (Helisys Corporation, 1998).

In addition, a so-called "burn-out layer" method was already proposed to handle the overlap zone generated by the part's horizontal area (Cohen, 1991). In this method, laser is used to cut entire layers of sheet material for the horizontal areas of the part by means of intensive fine crosshatch method to make the part and waste easily separated. There are two major disadvantages of this method. One is that the method can only be applied to the horizontal areas of the part, not on the part surface with a slope, especially not on the surfaces that are almost horizontal. The large contact area between the inclined surface and the waste makes de-cubing very difficult. The other disadvantage is that denser laser beams are also applied to the waste area that does not contact the part, which increases the working time and decreases the efficiency.

In light of the above disadvantages, a laser path planning of burn-out rule is proposed in this paper. The threshold value of the slope of the part is developed based on the parameters such as the sheet material thickness and diameter of laser beams. If the slope of the part falls below the threshold value it means that the overlap zone between the waste and the part is too large, which increases the difficulty of de-cubing. In this case, a dense laser path is designated to the overlap zone to burn out the binder. On the contrary, if the slope of the part exceeds the threshold value, the burning-out rule is not employed. The optimal distance between the two adjacent laser paths is calculated according to the thermomechanical-model of the burn-out zone. Experimental results indicate that the method increases the de-cubing efficiency and reduces the de-cubing time required.

\section{The binder's thermal property of LOM paper}

The study of adhesive burn-out rule proposed in this paper requires complete understanding of the qualities of the adhesive. The Thermogravimetric Analyzer TGA 2950 is used to analyze the adhesive used in LOM. The polymer material to be tested is placed on the TGA test platform. The test platform sends the material to an airtight heating chamber. As the temperature in the chamber rises gradually, the polymer material gradually releases different ingredients of its composition until all ingredients are released. As the material releases its ingredients along with the increase in temperature, the weight of the material decreases. Especially when the temperature reaches the onset decomposition temperature of a certain ingredient, all of a sudden, a significant percentage of weight is released. Thus, the weight percentage curve drops steeply at such an instant. At the same time, the first-order differential curve also experiences rapid changes. Figure 1 shows the characteristics of adhesive resin, in which the vertical axis denotes the variation 
Figure 1 The thermal property of the binder of LOM paper (LPH042) tested by thermogravimetric analyzer. The onset decomposition temperature is $235.44^{\circ} \mathrm{C}$
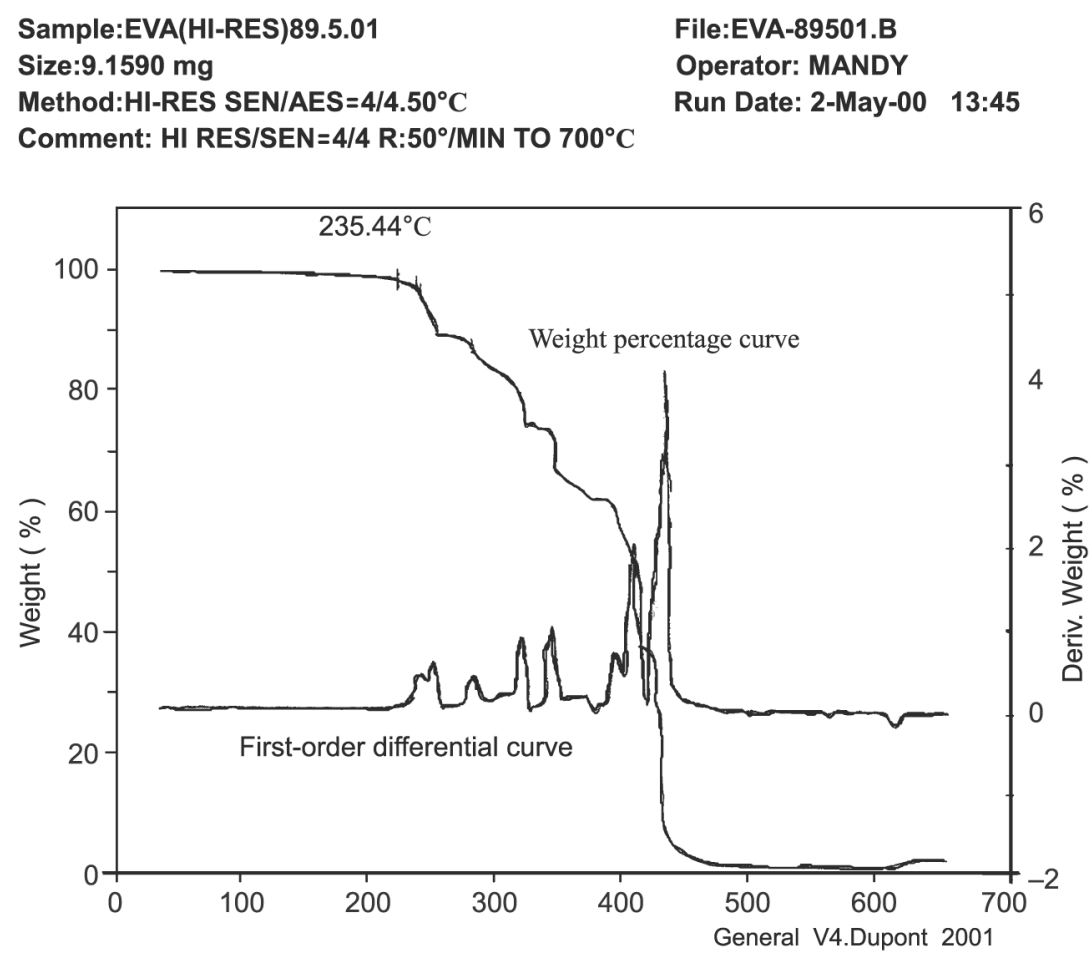

of weight percentage while the horizontal axis is the variation of temperature.

The test LOM paper adhesive is coded as LPH042, which is made of ethylene-vinyl acetate (EVA) copolymer. During the analysis via TGA, the weight percentage decreased dramatically as the temperature rose to $235.44^{\circ} \mathrm{C}$. The EVA did not completely decompose until the temperature reached $430^{\circ} \mathrm{C}$. In other words, the temperature of $235.44^{\circ} \mathrm{C}$ is the onset decomposition temperature of EVA, which is also an irreversible temperature. When the temperature reaches beyond this value, EVA decomposes and releases its ingredients. Later, if the temperature falls below this temperature, EVA no longer retains its adhesive function. Thus, the temperature of $235.44^{\circ} \mathrm{C}$ can be regarded as the decomposition temperature that determines whether the adhesive has burned out or not. As long as the temperature does not reach beyond its decomposition temperature, its adhesive qualities are not affected. From the perspective of adhesive burn-out, when the temperature generated by laser beams working on sheet material exceeds $235.44^{\circ} \mathrm{C}$, the sheet material is no longer sticky because the temperature has exceeded the decomposition temperature of EVA.

\section{The thermomechanical heated zone induced by laser cut}

\subsection{Thermomechanical analysis}

Before the heated zone of laser cut can be studied, a light gauge must measure the laser energy required to cut the sheet material under different working parameters.

The object to be measured is a $25 \mathrm{~W} \mathrm{CO}_{2}$ laser of LOM machine. The actual measurement results indicate that the laser energy is powerful enough to cut $\mathrm{LPH} 042^{\mathrm{TM}}$ paper under different duty cycles and ranges between $0.4 \mathrm{~W}(400 \mathrm{~mW})$ and $2 \mathrm{~W}$.

The proposed value is about 300 times of stereolithography laser energy capable of hardening the resin (between 3 and $31 \mathrm{~mW}$ ) (Chua et al., 1998). The measured energy matches their reported value. Thus, this data is used as the basis for development of the thermomechanical model.

The thermomechanical model resulting from laser cut is induced. During the process of laser cut, in addition to the laser working path where the sheet is cut, the area in the vicinity of the working path is also affected by the thermal energy, which burns out the adhesive. The problem of estimating the burn-out range can be simplified as the thermal effect of a laser beam moving at a fixed velocity (i.e. moving heat source) hitting 
on the sheet at an instant. Figure 2 shows a horizontal $(X-Y)$ sheet material undergoing laser cut. It is a control volume with the following boundary conditions.

\subsubsection{Boundary conditions}

The heat flux generated by the laser energy apparatus to the sheet to be cut via radiation can be calculated by the heat transfer coefficient, $h_{0}\left(\mathrm{~W} / \mathrm{m}^{2 \circ} \mathrm{C}^{1}\right)$. Under these circumstances, the boundaries of the heated zone generated by the laser beam, namely, $S_{1}, S_{2}, S_{3}, S_{4}$ and $S_{5}$ (as shown in Figure 2) can be considered as a convection boundary condition. Thus, the boundary condition of the contact area can be expressed as:

$$
K_{L} \frac{\partial T}{\partial y}=-h_{0}\left(T-T_{0}\right), \quad \text { on } \mathrm{S}_{1}
$$

where $K_{L}$ denotes the conductivity of the sheet material, which varies as the temperature changes. The value of $h_{0}$ is $20 \mathrm{~W} / \mathrm{m}^{2 \circ} \mathrm{C}^{1} . T_{0}$ denotes the temperature in the chamber and has a value of $40^{\circ} \mathrm{C}$. Since the platform is an enclosed space, it can be assumed to have a stable temperature.

Likewise, $S_{2}, S_{3}, S_{4}$ and $S_{5}$, as shown in equation (1), share the same boundary conditions.

\subsubsection{Governing equation}

Although LOM involves two kinds of different materials, namely, sheet material and adhesive, which have different thermal qualities, they are simplified into an anisotropic, but homogeneous continum. The thermal analysis considers only the thermal effect generated by the laser cut on sheet material, and ignores the heat generated by the thermal press roller (Sonmez and Hahn, 1998). This is because the purpose of the heat generated by the roller is to make the adhesive sticky, not to be burned out.

It is assumed that the heat source (i.e. laser spot) is evenly distributed and the heat loss along the $Z$ direction is ignored.

Figure 2 Thermomechanical model of laser path

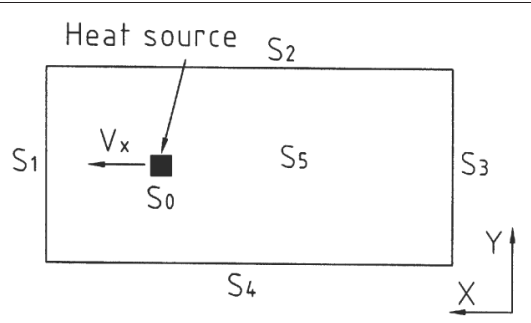

Since the value of $K_{z}$ is only one third of the values of $K_{x}$ and $K_{y}$, and the thermal resistance effect in the $Z$ direction, the analysis can be simplified into a 2D analysis (Sonmez and Hahn, 1998). Thus, the governing equation of energy equilibrium is considered as an Eulerian control volume (Brodkey and Hershey, 1988; Chua, 1994; Feygin et al., 1992; Sonmez and Hahn, 1998), as shown in equation (2), which is a transient conductivity model:

$$
\begin{aligned}
\mathrm{S}_{0}: \quad K_{x} \frac{\partial^{2} T}{\partial x^{2}} & +K_{y} \frac{\partial^{2} T}{\partial y^{2}} \\
& =\rho C_{p} V_{x} \frac{\partial T}{\partial x}+\rho C_{p} \frac{\partial T}{\partial t}
\end{aligned}
$$

where $K_{x}$ and $K_{y}$ denote the conductivity $\left(\mathrm{W} / \mathrm{m}^{\circ} \mathrm{C}\right)$ in the $X$ and $Y$ directions, respectively, $\rho$ denotes the density $\left(\mathrm{kg} / \mathrm{m}^{3}\right)$, $C_{p}$ the heat capacity $\left(\mathrm{J} / \mathrm{kg}^{\circ} \mathrm{C}\right)$ and $V_{x}$ the laser working velocity $(\mathrm{m} / \mathrm{s})$. Since the horizontal direction of $X$ - and $Y$ - axis of sheet material are investigated, we may consider that $K_{x}=$ $K_{y}=K_{L}$. Variations of thermal conductivity and specific heat with changes of the temperature are shown in Figure 3. The density of the material number LPH042 $2^{\mathrm{TM}}$ remains constant and is unrelated to temperature (Sonmez and Hahn, 1998).

\subsubsection{Solution}

The mesh structure and uniform rectangle elements shown in Figure 4 are used to solve

Figure 3 Heat capacity and conductivity of material LPH042 under different temperatures

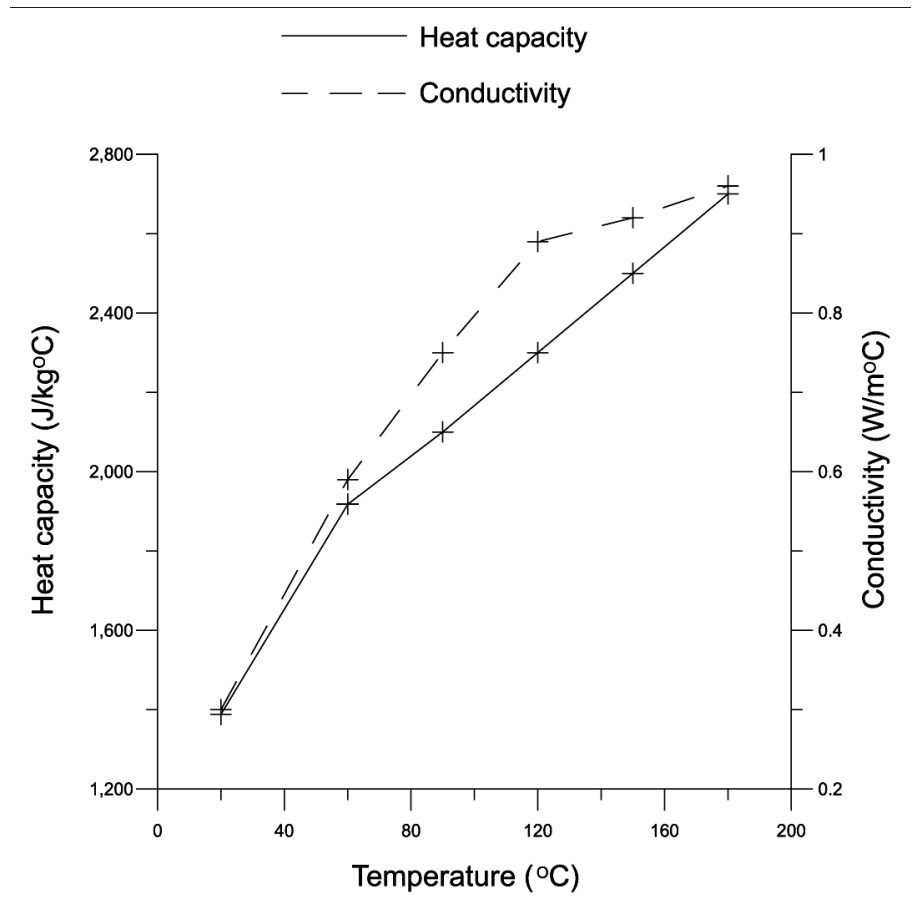


Figure 4 The corresponding relationship between temperature field and grid mesh of material LPH042 ${ }^{\mathrm{TM}}$. Laser spot size is $0.1 \times 0.1 \mathrm{~mm}^{2}$ area. Laser speed is $2.5 \mathrm{~cm} / \mathrm{s}$. Laser energy is $1 \mathrm{~W}$
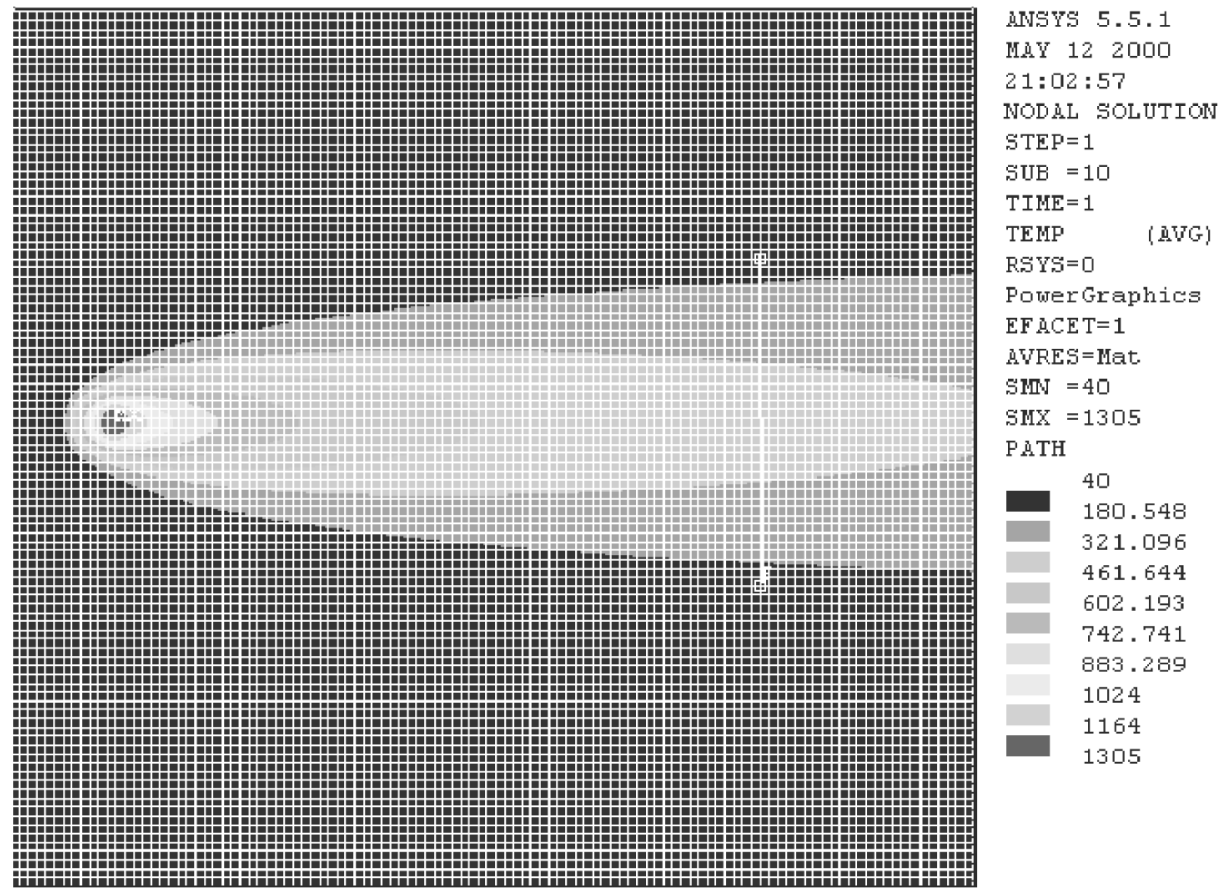

equation (2). Each element measures

$0.02 \mathrm{~mm}$ in length. The finite element method is used to calculate the temperature

distribution. Since $K_{x}, K_{y}$ and $C_{p}$ are functions of temperature, the exact solution cannot be derived. Thus, the software, ANSYS, is used to solve temperature distribution. The result is shown in Figure 4.

\subsection{Calculation of burn-out zone}

Figure 4 shows the corresponding relationship between the temperature field distribution and mesh structure. For the squared spot size $0.1 \times 0.1 \mathrm{~mm}$ in area, the highest temperature reaches around $1,305^{\circ} \mathrm{C}$. The temperature of the surrounding area decreases gradually to $40^{\circ} \mathrm{C}$. Since the laser beam velocity $V_{x}$ moves from right to left horizontally, the heated zone takes the shape of a comet. The purpose of the simulation is to investigate the adhesive effect inside the temperature field. Thus, attention is paid to the burn-out condition in the transverse side along the laser working path. According to the analysis of adhesive quality described in Section 2, the onset of decomposition temperature of EVA is $235.44^{\circ} \mathrm{C}$. Thus, the temperature of $235.44^{\circ} \mathrm{C}$ is considered as the burn-out temperature of the adhesive. Accordingly, the traverse area along the laser cutting path where its temperature exceeds $235.44^{\circ} \mathrm{C}$ is taken as burn-out zone. A correlation graph between the adhesive burn-out zone and temperature distribution is drawn and shown in Figure 5. The traverse area along the laser cutting path where its temperature exceeds $235.44^{\circ} \mathrm{C}$ can be expressed by the multiple value of laser beam diameter.

In the laser energy is $1 \mathrm{~W}$, working velocity is $2.5 \mathrm{~cm} / \mathrm{s}$ and laser cut of spot size is $0.1 \times$ $0.1 \mathrm{~mm}$. Under these conditions, the largest

Figure $\mathbf{5}$ The temperature profile induced by laser cut. Laser velocity is $2.5 \mathrm{~cm} / \mathrm{s}$ and energy is $1 \mathrm{~W}$

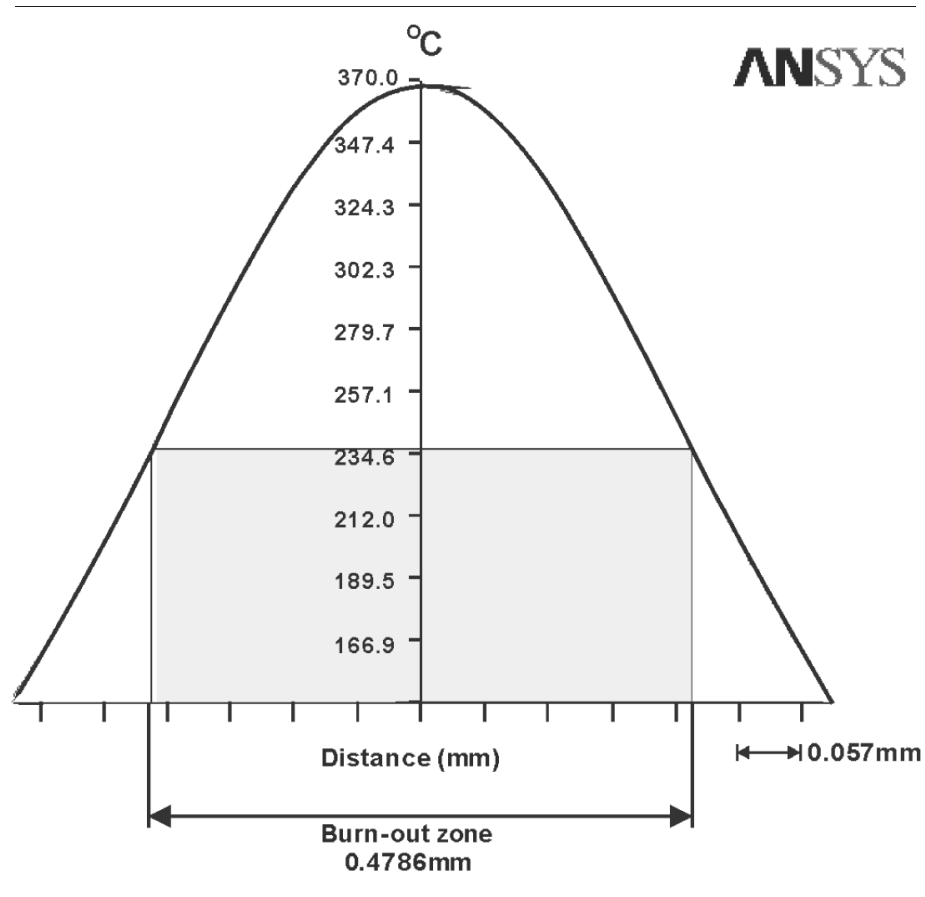


traverse width of the adhesive burn-out zone is $0.4786 \mathrm{~mm}$. This value is 4.786 times of the laser beam $(4.786 \times 0.1 \mathrm{~mm})$. Similarly, the effect of the heated zone generated by different laser energies, but a constant working velocity in order to observe the trend between the laser energy and burn-out zone can be induced. Figure 6 shows the variation as the laser energy shifts from 0.4 to $2 \mathrm{~W}$ under the constant working velocity of $2.5 \mathrm{~cm} / \mathrm{s}$. It can be seen that there is a linear relationship between the laser energy and the burn-out zone. When the laser energy increases, the burn-out zone also increases, and vice versa.

In the same way, the effect of different amounts of laser energy and different working velocities on the burn-out zone are analyzed systematically, and the results are shown in Figure 7. The following results can be observed. Given a constant laser energy of $0.4 \mathrm{~W}$, as the working velocity decreases from $V_{x}=5 \mathrm{~cm} / \mathrm{s}$ to $V_{x}=2.5 \mathrm{~cm} / \mathrm{s}$, the burn-out zone ranges from $1.0035 d$ to $1.8845 d$ where $d$ is the laser spot size. As the working velocity decreases further from $V_{x}=2.5 \mathrm{~cm} / \mathrm{s}$ to $V_{x}=1 \mathrm{~cm} / \mathrm{s}$, the expansion of the burn-out zone increases from $1.8845 d$ to $4.094 d$. Thus, from the perspective of burn-out, the lower the working velocity, the more significant is the increase of the burn-out zone.

Likewise, the greater the working energy, the larger is the burn-out zone, especially under the circumstances of low velocity and high power, which produce most satisfactory result. However, an overly strong working energy may cause other problems, such as

Figure 6 The relationship between burn-out zone and laser power under the laser velocity of $2.5 \mathrm{~cm} / \mathrm{s}$

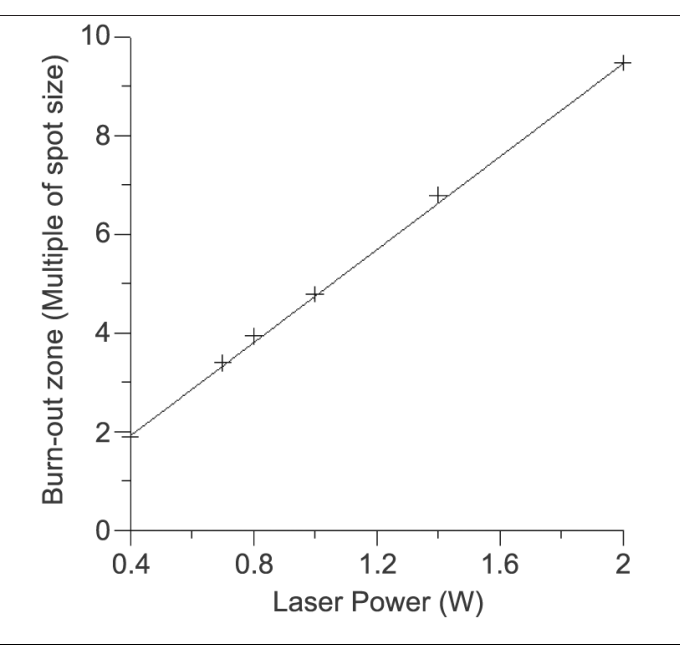

Figure 7 The relationship among working energy, velocity and multiples of spot size

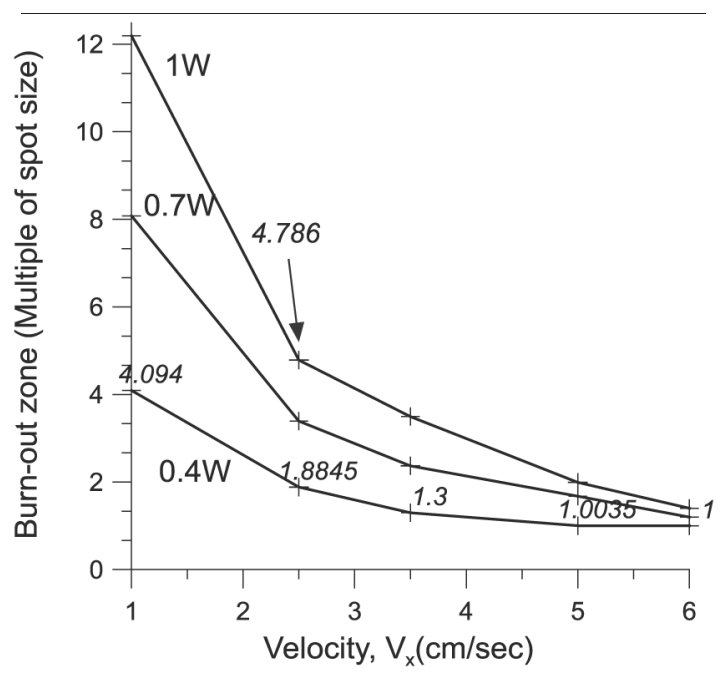

part deformation and poor precision.

Thus, the problem should not be handled purely from the burn-out viewpoint.

Based on the earlier discussion, consider the sheet working area impacted by the laser beam in an instant with a circular cross-section of diameter, $d$, then, given the constant values of the working energy and working velocity, the following equation can be concluded:

The burn out zone of heat affected

$$
=M \times d
$$

where $d$ is the spot size of laser cut, and $M$ depends on laser energy and working velocity.

\section{The effect of overlap zone}

\subsection{Determination of the part's inclination}

The STL file is used as the standard input file in the RP machine. Thus, the normal direction $(\xi)$ of STL and the part build orientation $(B)$ as shown in Figure 8 can be used to determine the inclination $(\eta)$ of every triangular face. It can be expressed as:

$$
\begin{gathered}
\vec{\xi} \cdot \vec{B}=D=|\vec{\xi}||\vec{B}| \cos \eta \\
\cos \eta=\frac{D}{|\vec{\xi}||\vec{B}|} .
\end{gathered}
$$

\subsection{The relationship between the part's inclination and overlap zone \\ Generally speaking, the size of the overlap zone between the waste and the part is the key factor affecting the difficulty of de-cubing.}


Figure 8 Schematic diagram of the part's inclination

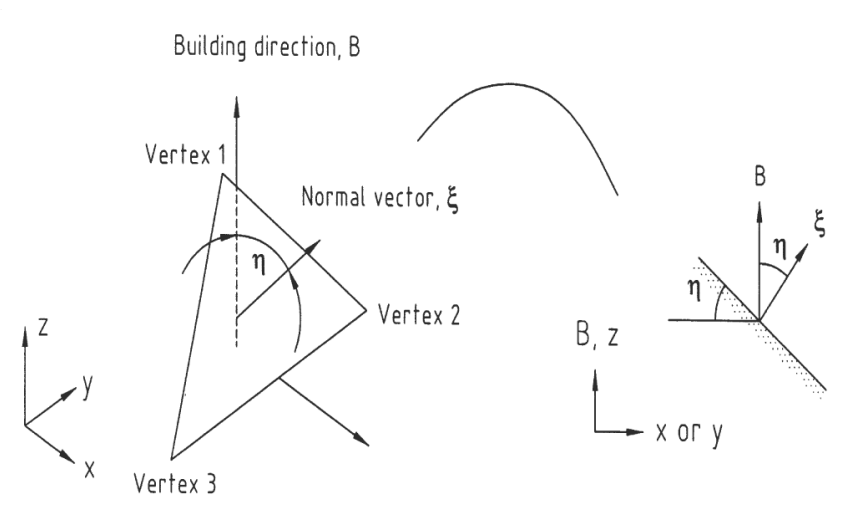

A large overlap zone increases the difficulty of de-cubing. As the overlap zone decreases, de-cubing becomes easier. The size of the overlap zone is closely related to the inclination of part contour and variation of sheet material thickness, as shown in Figure 9. The relation can be expressed by equation (5).

$$
\begin{array}{cc}
a=t \cdot \cot \eta, & \eta \neq 0 \\
a=\text { the width of triangle facet, } & \eta=0
\end{array}
$$

where $a$ denotes the width of the overlap zone between the waste and the part at every layer, $t$ denotes the thickness of sheet material, and $\eta$ denotes the inclination of the part's contour. Obviously, when the part's inclination is steeper or almost vertical, the overlap zone is smaller or even non-existent. When the part's inclination approaches zero or the horizontal level, the overlap zone is larger. If the inclination is horizontal $(\eta=0)$, the cot $\eta$ value is infinite, representing the largest overlap zone. In this case, the size of the overlap zone depends on the area of the triangular face. In other words, a horizontal

Figure 9 The relationship among the overlap zone, part's inclination and lamination thickness

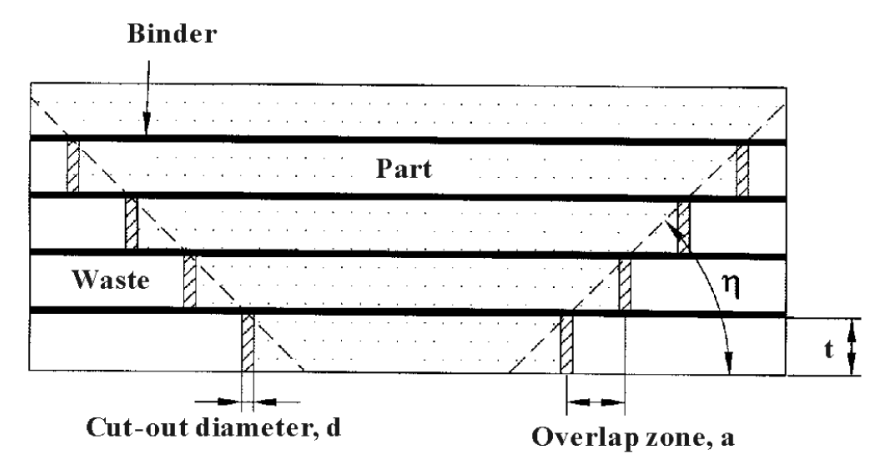

contour of the part is unfavorable for the de-cubing process. Thus, an experienced technician will try to make the contour vertical or almost vertical while deciding the optimal working direction in order to make de-cubing easier.

\subsection{Threshold value of the inclined surface}

Based on previous discussions of the adhesive burn-out zone caused by laser cut, it is assumed that the optimal burn-out method can be achieved when $M$ times of laser beam diameter $(M \times d)$ equals the width of the overlap zone $(a)$. The threshold value of the inclined surface $\left(\eta_{\mathrm{th}}\right)$ can then be derived. Equation (5) can be rewritten as:

$$
a=M \times d, \quad \eta_{\mathrm{th}}=\frac{\tan ^{-1}(t)}{(M \times d)}
$$

where $M$ denotes the multiplier of laser beam diameter as a result of the burn-out zone under a certain laser working energy and working velocity, as discussed in Section 3.2. In other words, when the part's inclination exceeds the value of $\eta_{\mathrm{th}}$, the de-cubing process is easy because of a smaller overlap zone between the waste and the part and the fact that the adhesive is already removed during the laser cut. There is no need for denser cuts. On the contrary, when the part's inclination is smaller than the value of $\eta_{\mathrm{th}}$, the resulted larger overlap zone makes de-cubing more difficult. Denser cuts are thus necessary to be employed.

Since the part's inclination $(\eta)$ and the sheet material thickness $(t)$ are important factors affecting the size of the overlap zone, they can be served as the basis for determining whether the burn-out rule is needed to be employed. According to this concept, the part's inclination threshold value can be derived for different values of thickness and laser beam diameter, as shown in Figure 10. Given the same sheet thickness, the larger the laser beam diameter, the smaller is the inclination threshold value required. On the contrary, the smaller the laser beam diameter, the larger is the inclination threshold value required. Similarly, given the same laser beam diameter, the thinner the sheet material, the smaller is the inclination threshold value required, and vice versa. To sum up, the smaller the inclination threshold value, the better the de-cubing process. This is because that a smaller threshold value implies a 
Figure 10 The threshold value of the inclined surface under different layer thickness $(t)$ and laser diameter $(d)$. Parameter: laser power $1 \mathrm{~W}$, working velocity $2.5 \mathrm{~cm} / \mathrm{s}$, multiples of spot size $4.786 d$

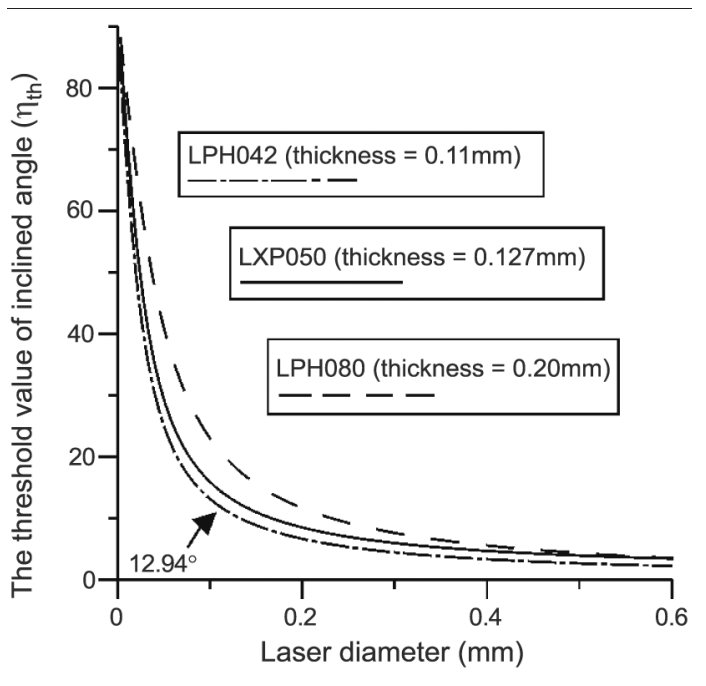

smaller chance for the burn-out process to be required, which reduces the working time. As shown in the trends in Figure 10, this objective can be reached by increasing the laser beam diameter and reducing the material thickness.

Let us consider a case for instance. Let the material be a piece of paper numbered LPH042 ${ }^{\mathrm{TM}}$ with a thickness of $0.11 \mathrm{~mm}$. The working parameters are as follows: laser diameter $(d)$ is $0.10 \mathrm{~mm}$; laser energy is $1 \mathrm{~W}$; working velocity is $2.5 \mathrm{~cm} / \mathrm{s}$. As shown in Figure 7 , the burn-out zone caused by laser cut is $4.786 d$. Incorporate the value of $M=4.786$ into equation (6) to derive the part inclination threshold value $\left(\eta_{\mathrm{th}}\right)$ of $12.94^{\circ}$. The threshold value can also be checked from Figure 10. Thus, if the inclination threshold is smaller than $12.94^{\circ}$, it is required to completely burn-out the adhesive in the overlap zone to facilitate de-cubing.

Meanwhile, the optimal laser cut distance is $4.786 d$. Although it seems to be simply the width of the burn-out zone, it can be used for determining the optimal distance between the laser path to burn-out the adhesive. When the laser cut distance is less than the value, the repeated and abundant laser path will waste much machining time. On the other hand, when the laser cut distance is larger than the value, the adhesive between the layers will stick to the waste material. Therefore, it will lead to unnecessary de-cubing time. That is why the optimal laser cut distance is $4.786 d$ under the above working parameters.

\section{Procedure of burn-out rule}

The procedure of the burn-out rule is briefly described as follows. Set the appropriate laser energy and working velocity, and then the optimal burn-out distance $(M \times d)$ is derived. The part's inclination threshold value $\left(\eta_{\mathrm{th}}\right)$ is determined based on the laser beam diameter $(d)$ and material thickness $(t)$. Then, calculate the overlap zone between the part and the waste if the slope of the part falls below the threshold value. Employ denser laser cuts to burn-out the adhesive based on the optimal burn-out distance in order to facilitate de-cubing. The detailed procedures are shown in Figure 11.

First, the STL file is retrieved and the laser beam diameter $(d)$ and the sheet material thickness used $(t)$ are input. Meanwhile, the proper part orientation $(\mathbf{B})$ is decided and the slicing operation from the smallest value on the $Z$ axis of the part is executed. The principle of slicing operation requires that an $Z$ axis plane intersects with all triangular faces that passes through the plane to derive the cross-section contour of the layer to be used in the working path. Hence, taking any triangular surface that passes through the $Z$ axis plane for slicing process, the inclination angle $(\eta)$ of the triangular surface is calculated. If the inclination angle is smaller than the threshold value $\left(\eta_{\mathrm{th}}\right)$, it means that there is an overlap zone between the triangular surface and the previous layer lamination. It is necessary to calculate the size of the overlap zone. After the size is derived, examine whether there is any other triangular surface passing through the $Z$ plane that has not been sliced. If the answer is yes, select the triangular surface for slicing process. If not, then all triangular surfaces passing through the $Z$ plane are already processed. If the inclination angle is larger than the threshold value $\left(\eta_{\mathrm{th}}\right)$, then there is no overlapping between the triangular surface and the previous layer lamination. In this case, it is still necessary to examine whether there are other triangular surfaces passing through the $Z$ plane that have not been sliced, and proceed slicing process for another triangular surface. After all triangular surfaces passing through the $Z$ plane have been processed, all these overlap zones are combined to form a closed curve and the burn-out rule inside the closed curve is employed. 
Figure 11 The flow chart of laser path planning of burn-out zone

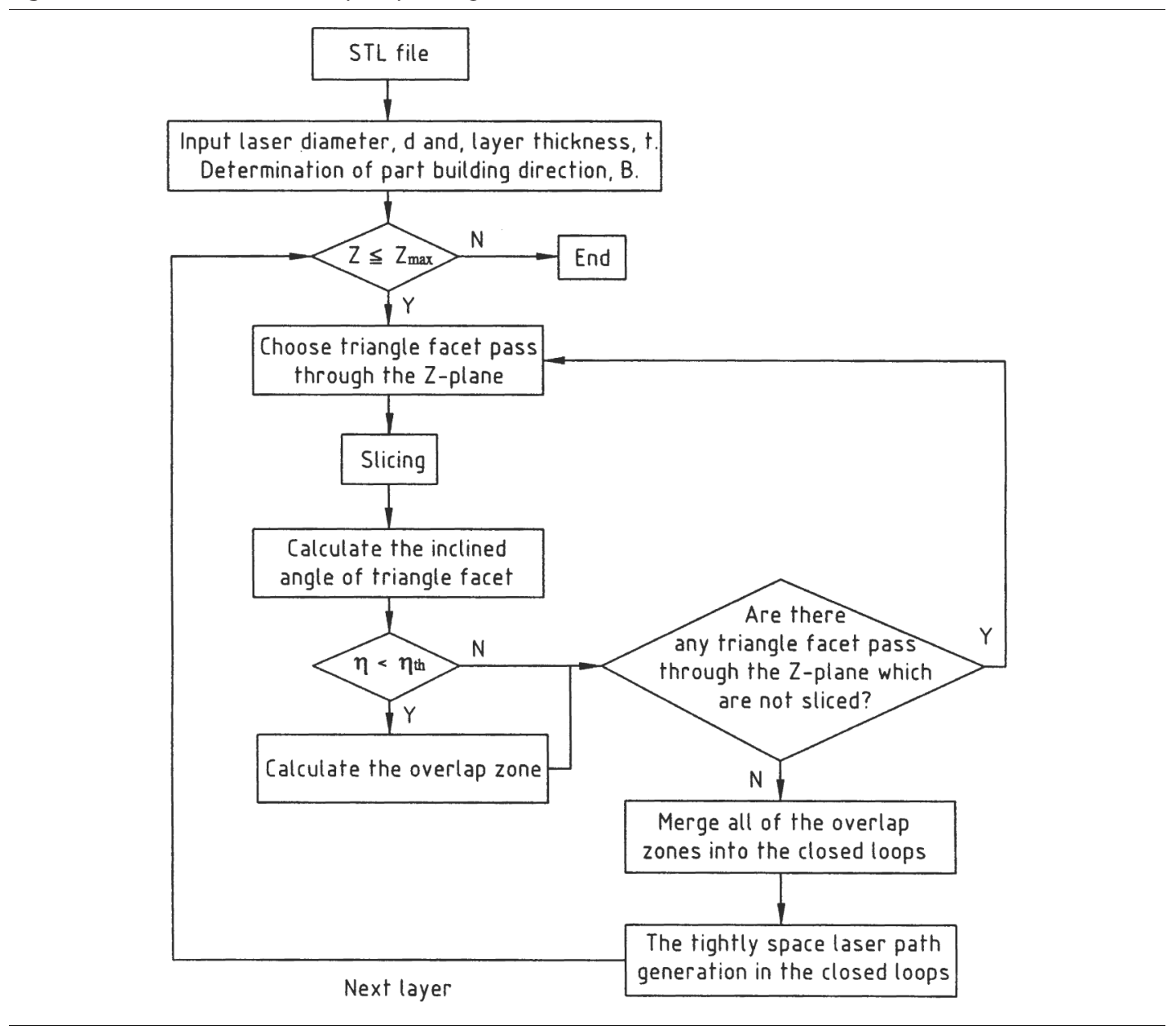

Here, the burn-out rule means the laser path processed in the closed curve based on the proposed optimal burn-out distance $(M \times d)$ between lasers, and then the adhesive in the zone will burn-out. Obviously, laser dark tracks will be left on the burn-out surfaces, as shown in Figure 12. Next, $Z$ axis is increased by an increment of one layer thickness and next layer is processed. The above steps are repeated until the maximum value of the part's $Z$ axis is reached.

\section{Example}

In order to demonstrate the validity of laser path planning of burn-out rule for LOM process, two different methods, namely general path and laser path planning of burn-out rule, are compared as shown in Figure 12. The material of the test part is paper numbered LPH042 ${ }^{\mathrm{TM}}$ with a thickness of $0.11 \mathrm{~mm}$. The working parameters are: laser diameter $(d)$ is $0.10 \mathrm{~mm}$; laser energy is $1 \mathrm{~W}$; working velocity is $2.5 \mathrm{~cm} / \mathrm{s}$. The part inclination threshold value $\left(\eta_{\mathrm{th}}\right)$ of $12.94^{\circ}$ is obtained from Figure 10 according to the above discussions.

The top drawing of Figure 12 is the front and top views of the test part, that has four surfaces less than $12.94^{\circ}$. The bottom left photo in Figure 12 is the part made by the laser path of burn-out rule. There are obvious burn-out tracks on the surfaces. The bottom right photo in Figure 12 is the part made by general path which is currently used by LOM process. As shown in Table I, the de-cubing time of general path is $25 \mathrm{~min}$, while the laser path of burn-out rule is $8 \mathrm{~min}$. The results indicate that the burn-out process saves 68 percent of the de-cubing time, significantly increasing the cutting efficiency.

\section{Conclusion}

A new method to improve the problem of de-cubing process for LOM is proposed in this paper. A novel laser path planning strategy is developed, it is based on 
Figure 12 Illustrate example
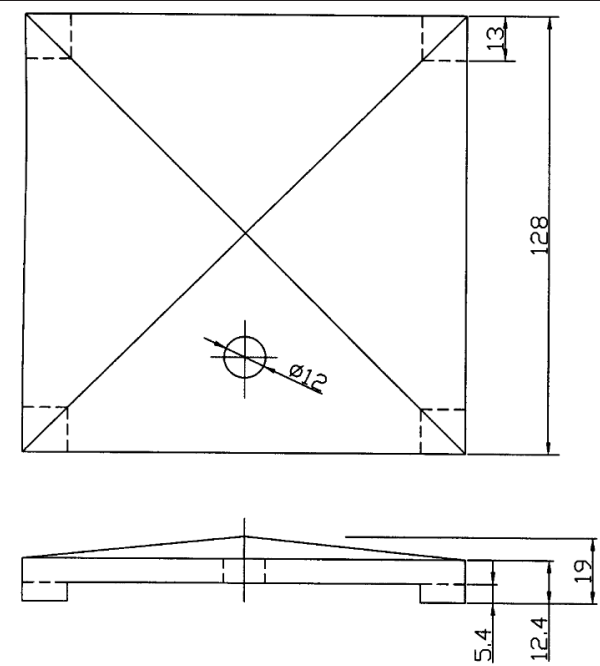

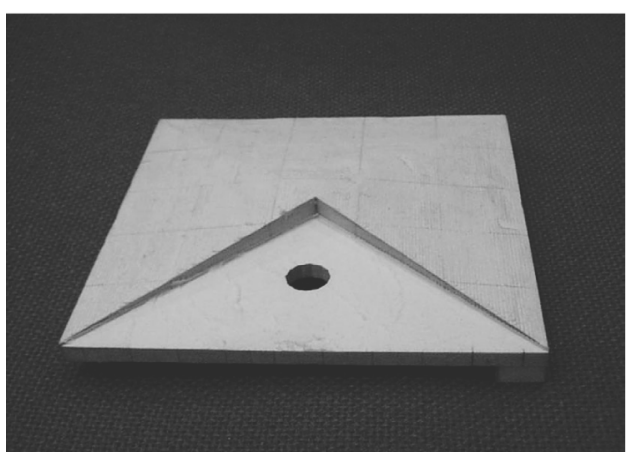

Table I Comparison of general path and laser path of burn-out rule

\begin{tabular}{lcc}
\hline & General path & Laser path of burn-out rule \\
\hline De-cubing time & $25 \mathrm{~min}$ & $8 \mathrm{~min}$ \\
\hline
\end{tabular}

the temperature of working area, being raised by the laser beams, reaches the decomposition temperature of the adhesive, the stickiness of the adhesive will be burn-out. Therefore, the burn-out rule is applied in the laser path planning based on the threshold value of part's slope. The following advantages of the burn-out rule proposed in this paper can be concluded as follows.

(1) The obvious burn-out tracks on the surfaces already treated by the burn-out rule makes it easier to distinguish between the waste and the part, and less likely to damage the part.

(2) The burn-out process significantly reduces the bonding strength between the part and the waste, making de-cubing much easier. The results indicate that the burn-out process saves 68 percent of the de-cubing time in this case, significantly increasing the cutting

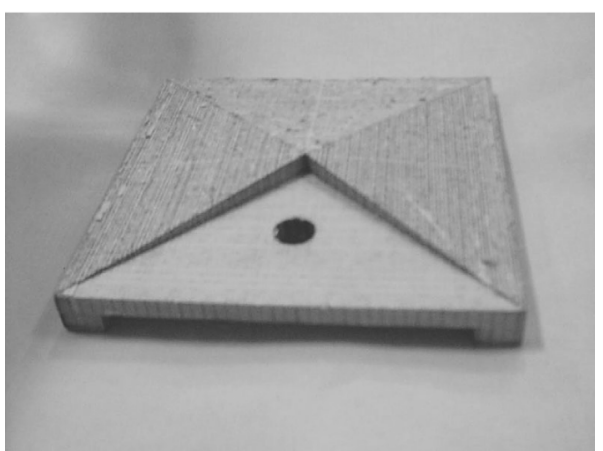

efficiency. In general, the ratio of surface area to volume can be used to predict the de-cubing time of the proposed laser path planning. While the ratio is high for complicated parts - sculpture, thin-wall and delicate parts, for example, the de-cubing time will be decreased apparently. It generally saves de-cubing time from 60 to 70 percent. While the ratio is low for simple part- thick-wall or large parts, the de-cubing time will be decreased slightly. In this case, it typically saves $20-30$ percent de-cubing time.

(3) From the perspective of the burn-out rule, the lower the cutting velocity, the greater the extent of increase in the burn-out zone. Besides, the greater the laser energy, the larger the burn-out zone and more significant is the burn-out effect. However, very strong laser energy may result in poor precision. Thus, it is suggested that the burn-out rule with ordinary power and low velocity should be used. 


\section{References}

Brodkey, R.S. and Hershey, H.C. (1988), Transport Phenomena, McGraw-Hill, NY, USA.

Cohen, A.L. (1991), "Technologyfocus: laminated object manufacturing", Rapid Prototyping Report, Vol. 1 No. 1, pp. 6-8.

Chua, C.K. (1994), "Three-dimensional rapid prototyping technologies and key development areas", Computing and Control Engineering Journal, Vol. 5 No. 4, pp. 200-6.

Chua, C.K., Chou, S.M. and Wong, T.S. (1998), "A study of the state-of-the-art rapid prototyping technologies", The International Journal of Advanced Manufacturing Technology, Vol. 14, pp. 146-52.

Feygin, M., Hsieh, B. and Melkanoff, M.A. (1992), "Laminated object manufacturing (LOM): a new tool in the CIM world", Eighth International PROLAMAT Conference, Man in CIM, Tokyo, Japan, Vol. B-3, pp. 457-64.

Helisys Corporation (1998), Value Analysis - Helisys vs KIRA.

Inui, E., Morita, S., Sugiyama, K. and Kawaguchi, N. (1994), "SAHP-A plain paper 3D printer/plotter process", The 5th International Conference on Rapid Prototyping, University of Dayton, Ohio, USA, pp. 15-26.
Klosterman D., Priore, B. and Chartoff, R. (1997a), "Laminated object manufacturing of polymer matrix composites", Rapid Prototyping Development Laboratory and Ohio Rapid Prototyping Process Development Consortium, University of Dayton, http://www.udri.udayton.edu/rpdl/Paper_SF2_97

Klosterman, D., Chartoff, R., Osborne, N. and Graves, G. (1997b), "Automated fabrication of monolithic and ceramic matrix composites via laminated object manufacturing (LOM)", Solid Freeform Fabrication Symposium Proceedings, University of Texas at Austin, pp. 537-49.

Klosterman, D., Chartoff, R., Osborne, N., Graves, G., Lightman, A. and Han, G. (1997c), "Laminated object manufacturing of advanced ceramics and composites", The 7th International Conference on Rapid Prototyping, March 31-April 3, University of Dayton and Stanford University, San Francisco, CA, pp. 43-50.

Klosterman, D., Chartoff, R., Priore, B., Osborne, N., Graves, G. and Lightman, A. (1996), "Structural composites via laminated object manufacturing", Solid Freeform Fabrication Symposium Proceedings, University of Texas at Austin, pp. 105-16.

Sonmez, F.O. and Hahn, H.T. (1998), "Thermomechanical analysis of the laminated object manufacturing (LOM) process", Rapid Prototyping Journal, Vol. 4 No. 1, pp. 26-36. 